\title{
Tip-structure effects on atomic force microscopy images
}

To cite this article: E Tekman and S Ciraci 1991 J. Phys.: Condens. Matter 32613

\section{Related content}
The electronic structure of transition metal interacting tip and sample and atomic force microscopy. II
$\mathrm{H}$ Ness and F Gautier
Theoretical modelling of STM, STS and AFM
D Drakova
Tip induced localized states in Scanning
Tunneling Microscopy
E Tekman and S Ciraci

View the article online for updates and enhancements.

\section{Recent citations}

- Chiara Manfredotti

M.M. Hadhoud and R.M. Atta

- Interpretation of "true atomic resolution" images of araphite (0001) in noncontact atomic force microscopy

A. Schwarz et al

\section{IOP ebooks}

Bringing you innovative digital publishing with leading voices to create your essential collection of books in STEM research. Start exploring the collection - download the first chapter of every title for free. 


\title{
Tip-structure effects on atomic force microscopy images
}

\author{
E Tekman and S Ciraci \\ Department of Physics, Bilkent University, Bilkent 06533 Ankara, Turkey
}

Received 22 August 1990

\begin{abstract}
We study the effects of tip structure on images in atomic force microscopy by using a periodic force field fitted to $a b$-initio force calculations. 'Ideal' images resolving the sample atoms can be obtained with stable and atomically sharp tips in the repulsive range. In the weakly attractive range protrusions may occur at locations different from the atomic positions. Multiatom tips usually yield distorted images in which only the size and the shape of the sample unit cell is conserved. Rotation of a blunt tip or a finite fiake lattice-matched to the sample causes stripes to form. Similar patterns can also appear for incommensurate sample-tip (or flake) systems.
\end{abstract}

The invention of atomic force microscopy (AFM) by Binnig, Quate and Gerber [1] constitutes a powerful tool for use either alone or combined with scanning tunnelling microscopy (STM) [2] in the analysis of surface structure [3]. In addition, it has a close bearing on STM operating at small tip-sample separation, where tip-sample interaction effects become crucial [4]. For example, the elastic deformation of the probing tip in the attractive force range modulates the tunnelling current and modifies the apparent corrugation obtained by STM. The transition from the tunnelling to the quantum ballistic regime in electron transport can be traced in the curve of the force or force gradient versus tip-sample distance $[2,4]$. The major advantage of AFM lies in the fact that the interpretation of the images does not require a detailed knowledge of the electronic structure of the sample. It was commonly assumed that the force variation is primarily determined by the repulsive interaction which is, in turn, proportional to the electronic charge density of the free surface, $\rho_{\mathrm{s}}\left(r_{0}\right)$. Atomic resolution has so far been achieved with repulsive forces of $10^{-6}$ to $10^{-8} \mathrm{~N}$ in the case of graphite [5]. There are, however, several issues which are controversial and require a deeper analysis. For instance, apart from the image of a honeycomb lattice, different image patterns (such as various stripes or 'every other atom' images) can also be obtained for graphite surfaces [6]. In particular, it is not obvious that AFM is sensitive enough to distinguish two different atomic sites in the (0001) plane. Moreover, strong repulsive forces measured [5] appear sufficient to induce irreversible (plastic) deformations [7-9]. Even if the outermost tip atom is within range of the repulsive force, the tip atoms at the back of the apex lie either in the strongly attractive or in decreasingly attractive force range. In addition, the sum of the weak attractive van der Waals forces being exerted on the atoms further away is uncorrugated but may be large owing to the forces' longer range. Therefore the repulsive forces acting on the atoms at the apex of the tip are always underestimated [10]. The situation becomes 
even more complicated when the deformation of the tip and the internal forces generated therein are taken into account.

In a recent study based on a frst principles calculation it was shown that the ion-ion repulsion dominates the force on the outermost tip atom in the repulsive and strongly attractive range $[4,10]$. This means that the tip images the sample ions if the force on the apex dominates the total tip force. The AFM images can only be related to the total charge density of the bare sample when the tip scans in the weak attractive range. In this range the potential barrier between the surface and tip is finite and the force corrugation is negligible [4]. The difference of one or two orders of magnitude between the measured tip force and that calculated for the outermost atom was explained by a thin contamination layer, or by a flake of graphite, which is dragged along with the tip [9,11]: A similar situation can also arise if the tip is flat $[9,10]$. Recent simulations by molecular dynamics, however, demonstrated that tips with several outermost atoms hinder resolution of the atoms and yield images that strongly depend on the structure and orientation of the tip [9]. The appearance of the stripes in some AFM images of graphite was attributed to a graphite flake which is rotated with respect to the graphite sample [7].

Clearly, the AFM images are strongly dependent on the shape and character of the tip, whereas the actual atomic structure of the tip itself is uncertain, and cannot easily be accessed experimentally. Currently, this seems to be the most serious limitation of AFM and it prevents us from a reliable simulation of the observed AFM images based on the calculations of force. In this report we investigate the effect of the shape of the tip on the AFM images. We consider various plausible tip structures which are typical to AFM, and perform a Fourier analysis [12] for the forces generated between these probing tips and the sample. The images constructed from these forces yield quite generic patterns. The analysis of the patterns allows us to draw conclusions of general validity and provides insight to the interpretation of images obtained from AFM. In this respect, the present study is complementary to our earlier work, which dealt with the microscopic aspects of the tip-sample interactions $[4,10]$, as well as to studies which used pair potentials $[8,9,13,14]$.

We consider a periodic force field, from which the force acting on a tip can be obtained. This is a reasonable approximation if the tip is prevented from inducing plastic deformations or hysteric displacements of atoms which irreversibly destroy the periodicity of the sample. Furthermore, we assume that the force is additive as in the case of forces derived from pair potentials $[8,14]$. This is valid if the tip is made from an inert material and if the form factors of atomic forces are estimated from a multiatom tip. We believe that deviations from the additivity can be tolerated as long as our objective is to reveal the effect of the tip structure rather than to perform a rigorous calculation of the tip force. This approximation allows us to extract the structure effects from the final force expression. The normal component of the force on a particular tip atom is given by

$$
F_{\downarrow}(x, y, z)=\sum_{h, k} \mathrm{e}^{2 \pi i(h x+k y)} f_{h k}(z)
$$

The lateral position of the tip atom is determined with respect to the lattice (and thus to the lattice vectors $R_{1}, R_{2}$ ) of the sample surface by $\rho=x R_{1}+y R_{2}$, and its height from the surface is $z$. The indices of the form factors, $f_{h k}$, specify the reciprocal lattice vector $G_{h k}=h G_{1}+k G_{2}$ from which they are derived. If we consider a tip of $N_{1, l} \times N_{2, l}$ unit 
Sample

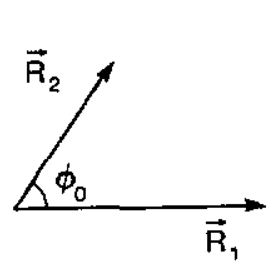

Tip

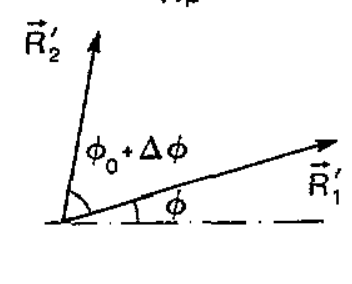

$$
\left|\vec{R}_{1}^{\prime}\right|=s_{1}\left|\vec{R}_{1}\right| ;\left|\vec{R}_{2}^{\prime}\right|=s_{2}\left|\vec{R}_{2}\right|
$$

Figure 1. Description of parameters relating tip structure to sample surface. $R$ and $\boldsymbol{R}^{\prime}$ are the Bravais lattice vectors for the sample surface and the tip, respectively.

cells in a given layer $l$, the perpendicular component of the total tip force is obtained by adding the individual atomic forces given by equation (1)

$$
F_{\perp}=\sum_{l} \sum_{h k} S_{N_{l, l}}\left(\psi_{1}\right) S_{N_{2 . l}}\left(\psi_{2}\right) \sum_{\mu \in l} \exp \left\{2 \pi \mathrm{i}\left[h\left(x_{l}+x_{\mu}\right)+k\left(y_{l}+y_{\mu}\right)\right]\right\} f_{\mu, h k}\left(z_{l}\right) \text {. }
$$

Here, $\mu$ denotes basis atoms in the tip unit cell in a given layer $l$. Their positions $\left(x_{\mu}, y_{\mu}\right.$, $z_{l}$ ) are defined relative to the vectors $R_{1}$ and $R_{2}$. The structural factors $S_{N_{1, l}}$ and $S_{N_{2} l}$ contain information regarding the Bravais vectors of the tip and sample unit cells and their relative orientation. The above expression can also be applied when the tip layers are not parallel to the sample surface. In this case, $S_{N_{1, l}}$ and $S_{N_{2, l}}$ disappear and the summation over layers is replaced by the summation over individual tip atoms.

The distortion of the 'ideal' images for multiatom tips can be analysed by investigating the dependence of $S_{N_{1, l}}$ and $S_{N_{2, l}}$ on the tip-sample geometry. The latter have the usual functional dependence, $S_{N}(x) \stackrel{=}{=} \sin (N \pi x) / \sin (\pi x)$, on their arguments. $\psi_{1}$ and $\psi_{2}$ are expressed in terms of the geometrical parameters as:

$$
\begin{gathered}
\psi_{1}=\boldsymbol{G}_{h k} \cdot \boldsymbol{R}_{1}^{\prime} / 2 \pi=s_{1}\left[h \cos \varphi+\left(k R_{1} / R_{2}-h \cos \varphi_{0}\right) \sin \varphi / \sin \varphi_{0}\right] \\
\psi_{2}=\boldsymbol{G}_{h k} \cdot \boldsymbol{R}_{2}^{\prime} / 2 \pi=s_{2}[k \cos (\varphi+\Delta \varphi) \\
\left.-\left(h R_{2} / R_{1}-k \cos \varphi_{0}\right) \sin (\varphi+\Delta \varphi) / \sin \varphi_{0}\right]
\end{gathered}
$$

where $s_{1}$ and $s_{2}$ are the ratios of the lengths of Bravais vectors of the tip and sample. $\varphi_{0}$ and $\left(\varphi_{0}+\Delta \varphi\right)$ are the angles defined by the sample and tip unit cells, respectively, and $\varphi$ is the rotation angle of the tip unit cell with respect to the sample unit cell. These structural parameters that appear in the present analysis are described in figure 1 . It is seen that the tip and sample structures enter into $S_{N_{1, l}}$ and $S_{N_{2, l}}$ in a complicated way. Nevertheless, it is possible to extract useful conclusions directly from equations (2) and (3) assuming that the tip unit cell contains only one basis atom, and thus, that all the effects causing the distortion of images are sought in $S_{N_{1, l}}$ and $S_{N_{2, l}}$. For example, $S_{1}(\psi)=1$ for all $\psi$ indicates that the AFM images obtained by an atomically sharp tip (with a single atom at its apex) are not distorted, as expected. Also, for any $N$ one finds $\left|S_{N}(n)\right|=N$ and $S_{N}(m / N)=0$ if $n$ is any integer and $m$ is an integer which is not a multiple of $N$. Hence, depending on the geometry, some Fourier components of the force field may stay undistorted while some may be totally depressed in the total force expression. On the other hand, the average total tip force (corresponding to $h=k=0$ ) is independent of the structure factors and always becomes equal to the average force for a 
single atom times the number of atoms in a given layer surnmed over $l$. Usually, the arguments $\psi$ will not be equal to an integer or to a rational number. An upper bound

$$
\left(\left|S_{N}(\psi)\right| / N\right)^{2}<\left[1+\tan ^{2}(\pi / N)\right] /\left[1+N^{2} \tan ^{2}(\pi / N)\right]
$$

with the condition that $\psi$ is not closer than $1 / N$ to an integer can be derived for the general case. For example, for $N \geqslant 3$ this value is smaller than 0.45 . This implies that for a blunt tip or flake with large $N_{1, l} \times N_{2, l}$ the average total tip force is multiplied by the number of atoms facing the sample at the same height, whereas the total force corrugation cannot be enhanced in the same way. Earlier, a similar situation was pointed out for the lateral conservative tip forces [15].

By considering special tip structures it is also possible to deduce some useful rules regarding to the distortion of images. First we consider the effect of the rotation of the tip with respect to the sample. For a linear tip $\left(N_{1, l}=1, N_{2, l} \neq 1\right)$, only $S_{N_{2}, l}$ is different from unity, providing weighted sums of the Fourier components; this will lead to lowering of the image symmetry, and formation of some zig-zag-like shapes. However, for a flake or blunt tip $\left(N_{1, l}, N_{2, l} \neq 1\right)$ both $S_{N_{1, l}}$ and $S_{N_{2, l}}$ will cause different scalings, and thus only some of the symmetrically equivalent Fourier components will contribute to the force and yield stripe-like features in the images. On the other hand, since $s_{1}$ or $s_{2}$ only enters in one of the structural factors $S_{N_{1, l}}$ or $S_{N_{2, l}}$ in equation (3), while the rotation angle $\varphi$ appears simultaneously, the elongation or contraction of the tip in one direction is more likely to induce some zig-zag-like distortions.

In what follows, we will construct AFM images from the expression given in equation (2), and illustrate the effects originating from the structure of the probing tip. To this end, we consider different tip-sample systems, i.e., graphite sample and aluminium tip, and aluminium sample and aluminium tip, for which the variations of the force on a single tip atom as a function of the tip-sample separation, $F_{1}(z)$ were obtained from $a b$ initio force calculations using the Hellman-Feynman theorem within the local density approximation $[4,10]$. The force curves calculated for individual tip atoms at the special positions above the sample surface (top ( $T$ ) and hollow (H) sites) are fitted to Morsetype pair potentials expressed by

$$
U(r)=U_{0}\left(\mathrm{e}^{-2\left(r-r_{0}\right) / a}-2 \mathrm{e}^{-\left(r-r_{0}\right) / a}\right)
$$

so an analytical form for $F_{\perp}(\boldsymbol{\rho}, z)$ is obtained from the gradient of the superposition of these potentials. Certainly, this Morse potential with two parameters does not provide enough freedom to obtain a rigorous fit and, in particular, underestimates the force corrugation between the $\mathrm{T}$ and $\mathrm{H}$ sites. However, it provides a simple analytical expression for the force curve to allow extrapolation over the sample unit cell and construction of the periodic force field. We believe that the force field obtained by this procedure is adequate to explain various geometry effects. The parameters of the Morse potential fitted to the force curves obtained from $a b$-initio calculations are $U_{0}=0.24 \mathrm{eV}$, $r_{0}=2.91 \AA$ and $a=0.88 \AA$ for the Al-AI system and $U_{0}=0.053 \mathrm{eV}, r_{0}=2.74 \AA$ and $a=0.42 \AA$ for the graphite-Al system.

Figures 2 and 3 illustrate the contour plots of the tip forces calculated for various tipsample systems, which can be compared with the images obtained in the constant-height mode of AFM. All the plots are calculated for the tip being in the repulsive force region. An atomically sharp tip or a blunt tip in registry with the $\mathrm{Al}(111)$ surface is able to resolve the atoms in the repulsive-force region (figure $2(a)$ ). This can be considered as an 'ideal' image. According to results of ab-initio force calculations [10], the force gradient 
(a)

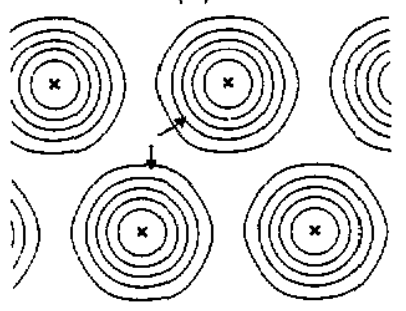

(b)

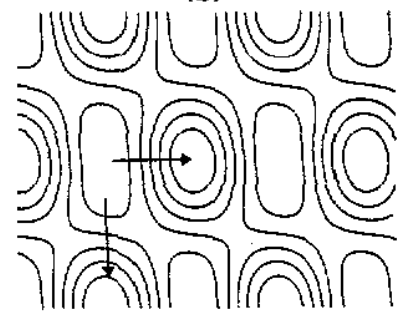

(c)

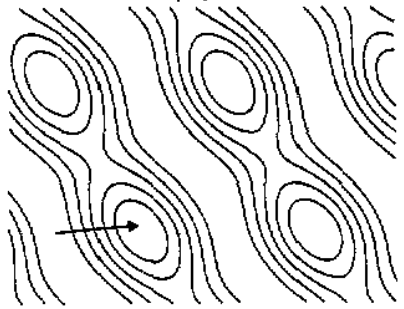

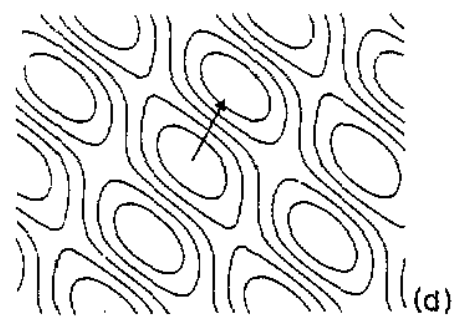

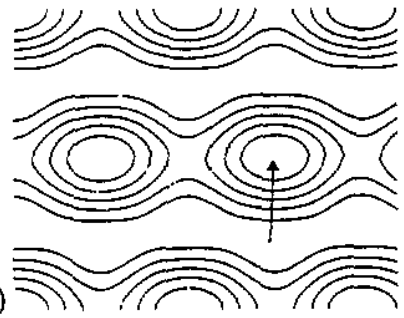

Figure 2. Contour plots of the tip forces at constant height $z=2 \AA$ for the Al(111) sampleAl tip system. The tip is $(a)$ a $(4 \times 4)$ flake in exact registry; $(b)$ a $(4 \times 1)$ linear tip rotated by $\varphi=10^{\circ} ;(c)$ a $(4 \times 4)$ flake rotated by $\varphi=10^{\circ} ;(d)$ a $(4 \times 4)$ flake contracted by $15 \%$ along the $R_{1}^{\prime}$ direction and $(e)$ a $(4 \times 4)$ flake with $\mathrm{Al}(100)$ structure $\left(\Delta \varphi=30^{\circ}\right)$. The arrows indicate the directions along which the force is increasing.

(a)

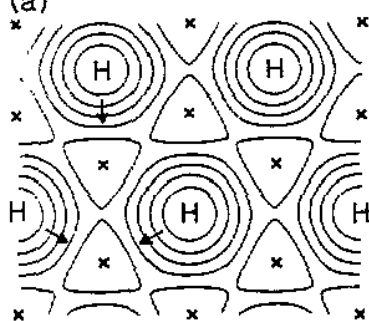

(c)

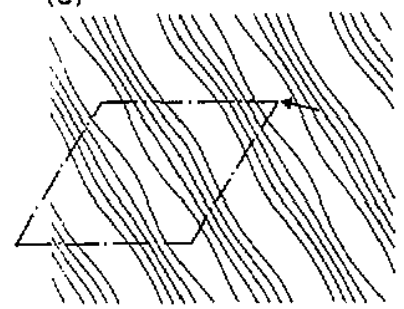

(b)

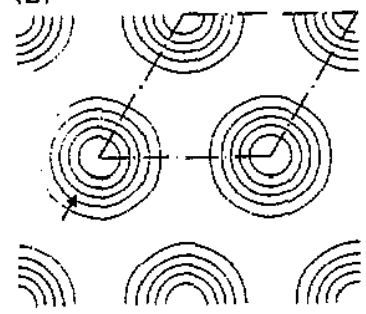

(d)

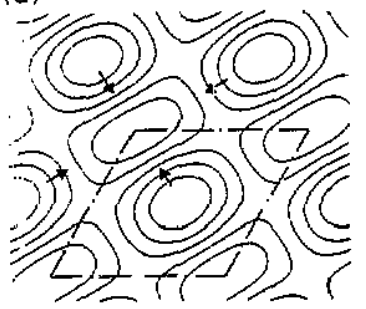

Figure 3. Contour plots of the tip forces at constant height $z=2.25 \AA$ for the graphite surface and $\mathrm{Al}$ tip. The tip is $(a)$ a single atom; $(b)$ a $(5 \times 5)$ flake in exact registry; $(c)$ a $(5 \times 5)$ flake in registry but rotated by $\varphi=10^{\circ}$ and $(d)$ a $(5 \times 5) \mathrm{Al}(111)$ flake.

vanishes at a smaller distance at the $H$ site, though for a larger force magnitude [10]. This seems to be a rather generic situation for many tip-sample systems, and occurs because of the ion-ion repulsion which happens to be significant at the $T$ site in the 
repulsive and strongly attractive force range. Therefore, the protrusions in figure $2(a)$ correspond to the atomic sites. In the weakly attractive range the force curves, $F_{\perp}(z)$, corresponding to the $\mathrm{H}$ and $\mathrm{T}$ sites may cross. In this case, the images obtained by a single atom tip cannot easily be identified with the positions of atoms in the sample surface $[4,10]$. Recently, Zhong and Tomanek [16] pointed out similar crossings for the adsorption energy of adsorbed Pd atoms on the basal surface of graphite, and hence for the potential energy determining the lateral conservative force related to the motion of the tip under a constant load.

If the tip has at least two outermost atoms arranged in a line but rotated by $\Delta \varphi$ relative to the sample, the ideal image starts to be distorted, and displays a zig-zag chain (figure $2(b)$ ). The rotation of the blunt tip or flake, which is otherwise in registry with the Al(111) sample, causes stripes to form in the images (figure 2(c)). A uniaxial distortion of the blunt tip, i.e. contraction or expansion in one direction, is enough to impose image distortion (figure 2(d)). A case observed frequently in AFM is that of a blunt tip or flake of different lattice structure giving rise to the formation of stripes (figure 2(e)). However, one notes that in all these such cases involving different distortion effects the surface unit cell of the sample is conserved in the AFM images. Similar features are also revealed for the images obtained from the graphite sample, which happens to be a central topic of AFM. As expected, all the atoms forming a honeycomb lattice can be seen if the tip is atomically sharp (figure $3(a)$ ). It seems that this is the only way to resolve all the atoms. Even if the tip or the flake is flat and has the same basis and same surface unit cell as the sample, and is parallel to the sample, only three protrusions out of six existing in a honeycomb lattice remain. This can be explained by the fact the tip force (which is a maximum when the tip and sample are in perfect registry) decreases when the tip is displaced by the basis vector. In this situation half of the tip atoms face the sample atoms, whereas the remaining half face the $\mathrm{H}$ site. This situation is illustrated in figure $3(b)$, in which the contour plots are obtained by using forces calculated for the $\mathrm{Al}$ tip and graphite sample. Since the behaviour of the force curve is quite generic in the repulsive region, our conclusion is independent of the details of the force curve. However, the above explanation may be no longer valid in the attractive force range for the reasons mentioned earlier. As seen in figure 3(c), even the 'every other atom' images experience dramatic distortion and display stripe patterns if the lattice-matched blunt tip or flake is rotated relative to the sample (i.e. $\varphi \neq 0$ ). A blunt Al tip, where the $2 \mathrm{D}$ lattice structures are completely uncorrelated, yields an 'every other atom'-like structure (figure $3(d)$ ). However, in all three panels (figure 3(b)-(d)) the 'every other atom' images are really the images of the surface unit cell, but have no direct relation to the sample atoms. Finally, we note that the distortions can be eliminated to some extent, and thus the resolution can be improved, if the tip is tilted to leave one fore front atom facing the sample.

In conclusion, we present an analysis of the AFM images, which reveals the effect of the tip structure. Our results suggest that:

(i) Atomic resolution can be obtained by an atomically sharp and stable tip in the repulsive force range. For some special cases, however, the hollow sites (instead of the atoms) in the sample surface may lead to larger deflections of the cantilever in the attractive force range.

(ii) Rotations of a lattice-matched blunt tip or a flake give rise to the formation of stripes in the images.

(iii) A tip having two or more atoms along one direction yields distorted images with zig-zags. 
(iv) A blunt tip or flake which is not lattice matched to the sample may yield both stripes and zig»zags.

This work was supported by the joint project agreement between IBM Zurich Research Laboratory and Bilkent University. Authors acknowledge stimulating discussions with Drs A Baratoff, I P Batra and E Stoll.

\section{References}

[1] Binnig G, Quate CF and Gerber Ch 1986 Phys. Rev. Lett. 56930

[2] Dürig U, Züger O and Pohl D W 1988 J. Microscopy 152 259; 1990 Phys. Rev. Lett. 65349

[3] For review see:

Heinzelmann H, Meyer E, Rudin H and Güntherodt H-J 1990 Basic Concepts and Applications of Scanning Tunneling Microscopy and Related Techniques ed R J Behm, N Garcia and H Rohrer (Deventer: Kluwer) p 443

[4] Ciraci S, Baratoff A and Batra I P 1990 Phys. Rev. B 427618

Ciraci 1990 Basic Concepts and Applications of Scanning Tunneling Microscopy and Related Techniques ed R J Behm, N Garcia and H Rohrer (Deventer; Kluwer) p 113

[5] Binnig G, Gerber Ch, Stoll E, Albrecht T R and Quate C F 1987 Europhys. Lett. 3 1281; 1987 Surf. Sci. 1891

Binnig G 1987 Phys. Scr. T 1953

[6] Marti O, Drake B, Gould S A C and Hansma P K 1988 J. Vac. Sci. Technol. A 6287

[7] Ciraci S and Batra I P 1987 Phys. Rev. B 366194

Batra I P and Ciraci S $1988 \mathrm{~J}$. Vac. Sci. Technol. A 6313

[8] Abraham F F, Batra I P and Ciraci S 1986 Phys. Rev, Lett. 601314

[9] Abraham F F and Batra I P 1989 Surf. Sci. 209 L125

[10] Ciraci S, Baratoff A and Batra I P 1990 Phys. Rev. B 412763

[11] Pethica J B 1986 Phys. Rev. Lett. 573235

Mamin H J, Ganz E, Abraham D W, Thomson R E and Clarke J 1986 Phys. Rev. B 349015

[12] Steele W A 1973 Surf. Sci. 36317

[13] Soler J M, Baro A M, Garcia N and Rohrer H 1986 Phys. Rev. Lett. 57444

[14] Gould S A C, Burke K and Hansma P K 1989 Phys. Rev. B 405363

[15] Mathew Mate C, McClelland G M, Erlandsson R and Chiang S 1987 Phys. Rev. Lett. 591942

[16] Zhong W and Tomanek D 1990 Phys. Rev. Lett. 643054 